\title{
LOS PRIMEROS FILÓSOFOS DE LA ACADEMIA LIMENSIS
}

\author{
The First Philosophers of the Limensis Academy
}

Milko H. Pretell García*

Recepción: 02-10-2018

Aceptación: 31-10-2018

\begin{abstract}
RESUMEN
La finalidad del siguiente trabajo es proporcionar datos en torno al desarrollo de la filosofía en los primeros años de funcionamiento de la universidad de San Marcos. Para ello se aborda, de una parte, la importancia de la Facultad de Artes y su conformación, y, por otra, los estudios comprometidos para asimilar los conocimientos filosóficos, lo mismo que los pasos para lograr convertirse en docentes. Cierran estas páginas la nómina de los primeros lectores de filosofía de la Universidad San Marcos.
\end{abstract}

\section{Palabras clave}

Filosofía Escolástica, Facultad de Artes, Universidad San Marcos, Virreinato, filósofos.

\begin{abstract}
The purpose of the following paper is to provide data on the development of philosophy in the first years of functioning of San Marcos University. To this end, the paper addresses, first, the development and importance of the Faculty of Arts; second, the course of studies designed for the study of philosophy; and, last, the required steps to become a lector. The paper closes with a list of the first philosophy lectores of San Marcos University.
\end{abstract}

\section{Keywords}

Scholastic Philosophy, Faculty of Arts, San Marcos University, Viceroyalty, philosophers.

* Coinvestigador del Proyecto Scholastica Colonialis-Perú desarrollado por la Pontificia Universidad Católica del Perú (PUCP): mhpretell@gmail.com 


\section{Preliminar}

La naciente sociedad y el desarrollo de las urbes en el recién creado Virreinato del Perú (1542) implicaron cubrir necesidades básicas diversas, entre ellas la formación técnica y profesional de su población. Esto último solo era posible a través del funcionamiento de un Estudio General o Universidad. Por ello el Cabildo de Lima solicitó al Rey la apertura de tal institución (1550), aunque -hasta donde se saberespondió a las iniciativas propuestas por los dominicos (1549).

$Y$ es que, en efecto, tanto el adoctrinamiento de la población aborigen como la formación de las nuevas generaciones obligaban a contar con centros de enseñanza. Lo primero hacía necesario un alto número de clérigos formados teológicamente y en lenguas autóctonas (lenguas índicas), para lo cual hubiese bastado que las órdenes clericales estableciesen sus centros de formación propios (Noviciados), sin embargo, también era necesario el reconocimiento legal de la formación profesional y la habilitación para su ejercicio, y ello solo era posible a través de la Universidad, la cual era la única responsable de otorgar los grados y títulos correspondientes.

Ahora bien, la formación profesional -prolongación de la formación medievalse ejecutaba en dos niveles tanto para el cuerpo secular como clerical: el nivel inferior correspondiente a los estudios humanistas (Gramática y Retórica) y de Artes (Lógica y Filosofía Natural), mientras que en el nivel superior se brindaban los estudios profesionales de Teología, Cánones, Leyes y Medicina. Y exactamente fue con estas cuatro Facultades superiores que nuestra universidad dio inicio a sus actividades académicas; sin embargo, la puerta de acceso a todas ellas le correspondía a la Facultad de Artes y los correspondientes estudios filosóficos.

Cabe, por lo tanto, preguntarse por cómo discurrieron los estudios filosóficos $y$, sobre todo, rescatar los nombres de quienes tuvieron en sus manos la potestad de la enseñanza de la filosofía, su difusión y debate. No obstante, son escasas las notas que existen en torno a los docentes y a su actividad académica, por lo cual aún quedarán muchas preguntas por contestar y lagunas en nuestra historia filosófica.

\section{La Facultad de Artes}

El jurista e historiador Luis Antonio Eguiguren ha sido quien remarcó la importancia de la Facultad de Artes al indicar que ella "en la universidad era la espina dorsal del sistema universitario" (Eguiguren, I, 1940, p. XLIII). No le faltaba razón, pues para acceder a los estudios profesionales como teólogos, abogados, matemáticos o médicos, era necesario tomar las lecciones de las cátedras impartidas en esta Facultad (Lógica y Filosofía Natural), como bien lo señala. Pero cabe indicar que ésta es una importancia heredada, pues nuestra universidad -lo mismo que el sistema de estudios superiores en general - se erige siguiendo el modelo de la universidad de Salamanca -la más importante en ese momento-, la misma que a su vez había tomado y mejorado el modelo de enseñanza de la universidad de París, conocido como modus parisiensis.

La universidad de París, por haber monopolizado la enseñanza de la teología -a instancias del papado-, imprimió un fuerte carácter filosófico a la Facultad de Artes, pues la Gramática (latín) y la Lógica (Dialéctica) fueron ampliamente desarrolladas en tanto que en el ideal de ciencia la teoría estaba por encima de lo 
práctico-experimental; se consideraba que a través del análisis silogístico se podía tener mayores asideros para encontrar la verdad. Por otra parte, la universidad de Salamanca importó a finales del siglo XV el modus parisiensis de mano de Francisco de Vitoria, planteando incluso modificaciones. Esta renovación fue de la mano con los restantes logros de la Corona española, permitiendo que Salamanca se convirtiese en el centro intelectual de su momento; así, a los estudios teológicos que habían logrado toda una renovación, se sumaron en importancia los estudios éticos-jurídicos, los estudios éticos-económicos y los estudios naturales ante la novedad del hallazgo del Nuevo Mundo, razón por la cual se entiende la adición de los estudios de Filosofía Natural (Física) a la curricula de la Facultad de Artes.

Teniendo en cuenta lo mencionado anteriormente se hace comprensible la importancia de la Facultad de Artes dentro del sistema educativo virreinal, se comprenderá también la estructura curricular que tuvo y los métodos de enseñanza empleados. Con respecto al modus Parisiensis se debe indicar que este estipulaba la gradación de temas a enseñarse para que sea un aprendizaje gradual, el dictado de las lecciones para que el alumno pueda luego repasar y la ejercitación del ars disputandi en eventos internos y públicos.

La universidad de Lima, lo mismo que los colegios y seminarios, solo permitían el inicio de los estudios de Artes luego de haber cursado los estudios de Gramática (latín), pues la lengua latina era el vehículo científico de aquel entonces. Los estudios se desarrollaban a lo largo de tres años y las lecciones se tomaban en dos horarios a lo largo del día, esto por el sistema educativo propio de la época, pues era necesario que los estudiantes dispusieran del mayor tiempo posible para sus lecciones. Así, las lecciones diurnas iniciaban por lo general a las 7 y culminaban a las 9 , luego seguían horas para el repaso; el segundo turno se iniciaba a las 4 p.m. y culminaba a las 5 p.m., empleándose luego algo del tiempo en realizar los repasos necesarios.

En teoría, el primer año abordaba los rudimentos de la Lógica (Dialéctica), el segundo se dedicaba a la Filosofía Natural (Física) y el tercer año permitía revisar cuestiones Metafísicas (Ontología). Sin embargo, en la práctica la ratio studiorum tanto de la Universidad como la de los jesuitas, lo mismo que de las restantes órdenes clericales que contaban con un centro de estudio, no aplicaron tal ordenación, pues los estudios metafísicos -materia más abstracta y compleja- fueron llevados en los estudios teológicos.

De los primeros años de la universidad no hay documentación que nos permitan determinar la estructura curricular. Se conoce la ratio studiorum de la universidad gracias a la Constitución de 1584, la cual señala la siguiente ordenación de temas a tratar. El primer año comprendía los estudios de las Summulae; se debía iniciar con el tratado sobre los términos y luego los parvos logicales por espacio de dos meses, después se empleaban dos meses para repasar los exponibles, esto es, el tratamiento de las proposiciones que parecen simples pero que en realidad son compuestas; se concluían las Súmulas con el estudio de los silogismos; a partir del octavo mes los alumnos se dedicaban a estudiar los predicables y los predicamentos. El segundo año estuvo dedicado a la Lógica, los siete primeros meses servían para revisar el Peri hermenias o De interpretatione, luego se proseguían con los restantes textos lógicos de Aristóteles; lamentablemente 
no hay mayores datos, pero bien podrían ser los Analíticos Segundos que se ocupan de la demostación científica. El último año de estudios comprendía la lectura de los Físicos (ocho libros) durante los primeros seis meses, lo restante era empleado en la lectura del De generatione et corruptione, así como el De coelo. Como se puede apreciar, la universidad puso énfasis en los estudios lógicos - por ende, comprometió a la Filosofía-, pues estos emplearon dos años para su aprendizaje.

\section{El oficio de Filósofo}

El título de "Filósofo" no fue empleado para designar a quienes culminaban los estudios de Arte. El título, ampliamente empleado durante la Edad Media, estuvo reservado para designar a Aristóteles, a quien propiamente se le designaba como "El Filósofo". En realidad, quienes cursaban los tres años de estudios en la Facultad de Artes solo obtenían los grados y títulos tras una serie de actividades que demostrasen el grado suficiente de preparación para poner en ejercicio los conocimientos adquiridos, esto es, para disputar sobre cuestión cualquiera y, claro está, también para enseñar.

Tenemos, por una parte, el grado de Bachiller para quienes no solamente asistieron a las lecciones brindadas, sino a quienes participaron en las disputas sabatinas que se organizaban de manera interna. Por otra parte, para convertirse en Licenciado en Artes, según la Constitución de 1571, era necesario que defendiera seis conclusiones de Filosofía Natural y Lógica magna y parva en el transcurso de un año, además para obtener el grado debía tener de una conclusión de Filosofía Natural al igual que leer una lección de algún tema extraído por azar de un texto de Filosofía (con solo un día de antelación), respondiendo las objeciones y/o preguntas de los maestros presentes. Esta lección debía darse en una hora.

Ahora bien, para acceder a la enseñanza era necesario participar en una junta de disputa denominada "oposición", es decir, era necesario un examen público que haga patente la idoneidad del futuro docente, tanto en cuanto a sus conocimientos como en la defensa de una conclusión establecida frente a las objeciones de los doctores oyentes. De este tipo de certámenes queda un registro, no de la universidad de San Marcos sino de la universidad del Cusco del siglo XVIII; registro que puntualiza los puntos a debatirse como: "de los quales escogió el primer punto y asentó por Conclusio: quod De potentia Dei absoluta duo corpora possunt esse in eodem loco" (Valcárcel, 1953, p. 40).

La labor docente implicaba, por la asimilación del modus parisiensis, servirse de un texto de ayuda para poder emprender las lecciones, esto es, explicar y/o comentar las ideas planteadas frente a los temas propuestos por el autor del texto, lo mismo que las objeciones presentadas. Todo lo cual debía ser llevado con las pausas correspondientes para que los alumnos pudiesen tomar las notas necesarias. Así tenemos, por ejemplo, que en los primeros años de funcionamiento de la universidad se empleó el texto de Domingo de Soto para el curso de Lógica. Igualmente, se conoce que la obra de Fray Alonso de la Veracruz también fue empleada al interior de algunos claustros clericales en el proceso de enseñanza de la Lógica. Por otra parte, tenemos también la elaboración y propuesta de textos "oficiales" para ser empleados localmente. Y son estos textos los que llaman potencialmente más la atención, pues en ella encontramos propuestas propias de nuestros filósofos que debaten 
de manera directa con sus pares europeos y con el mayor rigor técnico, pues con ello se deja por sentado que "poderoso es Dios para hacer surgir hijos a Abraham de las piedras peruanas".

\section{Los primeros filósofos de la Academia Limensis}

Es poco lo que se conoce respecto a quienes cumplieron con la labor de enseñanza de la Filosofía. Existe material disperso que necesita ser sistematizado en lo referente a nuestros primeros filósofos. Y, si bien L. A. Eguiguren nos ha facilitado una primera nómina de los 1 ectores de Artes y Filosofía en San Marcos, esta sigue siendo inicial y fragmentaria. Esto debido a que -por lo que se conoce hasta la fecha- no han sido los lectores de San Marcos quienes redactaron los Cursus philosophicus conocidos y que han logrado sobrevivir hasta nuestros días, lo cual les otorgaría con mérito ser denominados "filósofos"; los lectores que redactaron amplios Cursus han pertenecido a los colegios de los franciscanos, dominicos, agustinos y jesuitas.

Por otra parte, vale la pena implementar una división inicial en torno a la aparición de nuestros filósofos ${ }^{1}$. La primera que se nos viene en mente es la división por órdenes clericales; sin embargo, esta dejaría de lado el aspecto diacrónico de la evolución de los planteamientos filosóficos. La segunda tentativa que surge es la clasificación por orientación doctrinal; no obstante, en la medida que no hay estudios puntuales del quehacer filosófico de los pocos autores de textos y mucho menos existen obras de los que aparecen en la lista, sería un simple inventar. La tercera posibilidad, que por ahora se torna más congruente, es considerar la sucesión generacional. Así tendríamos como clasificación inicial una primera generación (15401590) de filósofos europeos que se convierten en los primeros maestros de las recién inauguradas aulas de los distintos claustros académicos. La segunda generación (1590 1640) comprendería principalmente a los primeros descendientes de los conquistadores tanto criollos como mestizos. Una tercera generación (1640 1700) asumirá las críticas al sistema escolástico fruto del Humanismo y la Reforma. La cuarta generación (17001767) es la que tiene que habérselas con el avance de la Filosofía Moderna y de las Ciencias (en sentido moderno), sin querer desprenderse del todo del modelo escolástico de exposición (empleo del silogismo). Finalmente, la quinta generación (1767-1821) que enfrenta las reformas educativas orientadas por el espíritu ilustrado y que culmina con nuestra independencia.

Ahora bien, la siguiente relación se reduce al claustro universitario, pues el correspondiente a los Colegios, Seminarios y Noviciados aún está en proceso de elaboración, pues hay mucha documentación dispersa y mínima al respecto. Veamos entonces quienes fueron los primeros maestros filósofos en el primer siglo de existencia de la Academia limensis.

(1) Martín Ugalde, lector seglar (1550¿1560?). ${ }^{2}$

1 Es interesante la división de tres etapas propuesta por Daniel Valcárcel, aunque está enfocada propiamente en el sistema educativo como tal. Cf. Valcárcel, D. (1968). Historia de la Educación Colonial. Lima: Universo.

2 Eguiguren, L. A. (1951). La universidad Nacional Mayor de San Marcos. IV Centenario de la Fundación de la Universidad Real y Pontificia y de su vigorosa continuidad histórica. Lima: Imprenta Santa María, p. 34; Eguiguren, L. A. (1951). La universidad en el siglo XVI, volumen I, narración. Lima: Imprenta Santa María, p. 56; en adelante: Universidad XVI. 
(2) Fray Miguel Montalvo OP (1550¿1563?). ${ }^{3}$

(3) Gaspar de Meneses (1565-1573), médico seglar. ${ }^{4}$

(4) Fray José Miguel de Adrián OP (1560¿11572?). ${ }^{5}$

(5) Jerónimo López Guarnido (1570-¿’?), médico español, natural de Sevilla. ${ }^{6}$

(6) Fray Pedro de Miranda OP (1573-1578). ${ }^{7}$

(7) Gerónimo Enríquez (1573). ${ }^{8}$

(8) Antonio Sánchez Renedo (1576 1581), médico de la universidad de Granada. ${ }^{9}$

(9) Fray Nicolás de Ovalle OM (15771586), discípulo del mercedario español Francisco Zumel. ${ }^{10}$

(10) Juan de Velásquez de Ovando (1580 $1583,1587) .{ }^{11}$

(1 1) Diego de Ávila Briceño (1593), natural de Huánuco, asumió la cátedra en 1576.
(12) Juan Velásquez de Ovando (15801587), empleaba la obra de Fray Domingo de Soto. ${ }^{12}$

(13) Diego de Ávila Briceño (1586 1592). ${ }^{13}$

(14) Juan de Obregón (1588), estudió en San Marcos. ${ }^{14}$

(15) Alonso de Abreu (1589-1591). ${ }^{15}$

(16) Carlos Marcelo Corne (1588 1598), criollo natural de Trujillo. ${ }^{16}$

(17) Fray Manuel de Figueroa y Miranda (1592-1594). ${ }^{17}$

(18) Gaspar de Centurión y Espinola (15921595), natural de Sevilla. ${ }^{18}$

(19) Diego de Ramírez de Cepeda (15951596), natural de Lima. ${ }^{19}$

(20) Diego de Hojeda OP (1595-1596), lector sustituto, natural de Sevilla. ${ }^{20}$

(21) Lorenzo López Barriales, sustituyó a Marcelo Corne por cuatro meses (1597). ${ }^{21}$

3 Eguiguren, L. A. (1939). Alma Mater. Lima: Imprenta Torres Aguirre, p. 116.

4 Eguiguren, Alma Mater, p. 250. Eguiguren, Universidad XVI, I, vol. 1, p. 456.

5 Eguiguren, Alma Mater, 1939. p. 160.

6 Eguiguren, Alma Mater, 300, 331; Universidad XVI, I, vol. 1, 475.

7 Eguiguren, Alma Mater, 259, 278; Universidad XVI, I, vol. 1, 467.

8 Eguiguren, Luis A. (1912). Catálogo histórico del claustro de la Universidad de San Marcos 1576-1800. Lima: Imprenta El Progreso Editorial. Córdoba Salinas, Buenaventura, Memorial de las historias del Nuevo Mundo Peru. Lima: Geromino de Contreras, 1631, edición s/f.; reproducción parcial en: Glave Testino, Luis M. Colección Pensamiento Educativo Peruano. Volúmen 2: Entre la sumisión y la libertad, siglos XVII-XVIII. Lima: Derrama Magisterial, 2013, p. 126. De ahora en adelante Glave, CPEP-II.

9 Eguiguren, Universidad XVII, I, vol. 2, 869,878,881, 901-2.

10 Eguiguren, Universidad XVII, I, vol. 2, 870, 888; Eguiguren, Luis A., Diccionario Histórico Cronológico de la Real y Pontificia Universidad San Marcos. Lima: Imprenta Torres Aguirre, tomo I, 1940, p. 327. En adelante: Eguiguren, DHC I.

11 Eguiguren, DHC I, 297; Universidad XVII, I, vol. 1, 525-26.

12 Eguiguren, DHC I, 297; Universidad XVII, I, vol. 1, 525-26.

13 Eguiguren, Universidad XVII, I, vol. 2, 934, 946; DHC, I, 237,682.

14 Eguiguren, Universidad XVII, I, vol. 2, 938.

15 Eguiguren, Universidad XVII, I, vol. 2, 938-39, 942.

16 Eguiguren, Universidad XVII, I, vol. 2, 939, 941, 960.

17 Eguiguren, Universidad XVII, I, vol. 2, 943, 947.

18 Eguiguren, DHC I, CCLXXXVIII; Universidad XVII, I, vol. 2, 946.

19 Eguiguren, Universidad XVII, I, vol. 2, 960.

20 Eguiguren, Alma Mater, 1939, p. 301; DHC, I, CCLXXVII-VIII.

21 Eguiguren, Universidad XVII, I, vol. 2, 966. 
(22) P. Andrés Hernández SJ (1599), cátedra supernumeraria. ${ }^{22}$

(23) Diego Ramírez de Cepeda (1599). ${ }^{23}$

(24) P. Cristóbal García SJ (¿̇?), cátedra supernumeraria. ${ }^{24}$

(25) Bachiller Gabriel de Sandi (1614). ${ }^{25}$

(26) Francisco de Godoy (1616). ${ }^{26}$

(27) Diego de Encinas Cañizares de Misque (1617), natural de Lima. ${ }^{27}$

(28) P. Francisco de Aguayo SJ (1620), cátedra supernumeraria. ${ }^{28}$

(29) Manuel de Esquivel Salcedo (1620 1634), primera Cátedra de Artes. ${ }^{29}$

(30) Pedro de Ortega y Sotomayor (¿16231628?), obtuvo la cátedra siendo colegial del Real de San Martín a la edad de 19 años y la regentó por espacio de seis años. ${ }^{30}$

\section{(31) Fray Gonzalo Tenorio OFM (1624). ${ }^{31}$}

(32) Francisco Tenorio (¿?), hermano del anterior. $^{32}$
(33) P. Fernando de Avendaño (1626 1629).

(34) Fray Juan de Ribera OSA (1627-1631), natural de Pisco, segunda Cátedra de Artes..$^{33}$

(35) Martín de Velasco y Molina (¿?) natural de España, estudió en el Colegio San Martín. ${ }^{34}$

(36) Andrés García de Zurita (1627), natural de Sevilla. ${ }^{35}$

(37) Diego López de Vergara y Aguilar (hasta 1631), natural de Lima, tercera Cátedra de Artes, se cerró su cátedra por ausencia de alumnos. ${ }^{36}$

(38) Sancho Pardo de Figueroa Bermúdez y Cárdenas (¿ं?), natural de Lima. ${ }^{37}$

(39) Fray Francisco de la Carrera OM (¿’). ${ }^{38}$

(40) Juan Jiménez de Montalvo (¿’?), natural de Lima. ${ }^{39}$

(41) Joseph de los Ríos (¿’?. ${ }^{40}$

(42) Juan de Utrilla (1647), natural de Lima, primera cátedra de Artes. ${ }^{41}$

22 Eguiguren, Catálogo, 1912, 46.

23 Eguiguren, DHC I, 993, 1046.

24 Eguiguren, Catálogo, 1912, 46.

25 Eguiguren, DHC I, 995.

26 Eguiguren, DHC I, 995.

27 Eguiguren, DHC I, 998.

28 Eguiguren, DHC I, 1001, 1005.

29 Eguiguren, DHC I, 1014,1017; Glave, CPEP-II, p. 126

30 Eguiguren, Catálogo, 1912, 47; Eguiguren, DHC, I, 891; Mendiburu, Manuel, Diccionario histórico biográfico del Perú. Segunda edición con adiciones y notas bibliográficas publicada por Evaristo San Cristoval. Lima: Imprenta Gil, 1934, tm. VIII, p. 273.

31 Eguiguren, Catálogo, 1912, 46

32 Eguiguren, Catálogo, 1912, 46

33 Eguiguren, DHC, I, 1013; Glave, CPEP-II, p. 127.

34 Eguiguren, Catálogo, 1912, 46.

35 Eguiguren, Catálogo, 1912, 46; Eguiguren, DHC, I, 820.

36 Eguiguren, DHC, I, 1012; Glave, CPEP-II, p. 127.

37 Eguiguren, DHC, I, 834

38 Eguiguren, Catálogo, 1912, 46.

39 Eguiguren, Catálogo, 1912, 46.

40 Eguiguren, Catálogo, 1912, 46.

41 Eguiguren, DHC, I, 28. 
(43) Fray Pedro de Córdova y Recalde OSA (1647), segunda cátedra de Artes. ${ }^{42}$

(44) Fray Juan Mezía de Mendoza OFM (1647), natural de Pisco, tercera cátedra de Artes. ${ }^{43}$

(45) Juan Morales y Valverde (¿'?), chantre de Lima. ${ }^{44}$

(46) Diego Josef de Salazar (¿’), de quien aún sobrevive un manuscrito sobre Metafísica. ${ }^{45}$

(47) Fray Sebastián de Almoguera y Pastrana OM (¿ं?). ${ }^{46}$

(48) Bartolomé de Velarde (¿’), natural de Arequipa. ${ }^{47}$

\section{REFERENCIAS}

Barreda y Laos, F. (1964). Vida Intelectual del Virreinato del Perú. 3ra ed. Lima: Universidad Nacional Mayor de San Marcos

Eguiguren, L. A. (1912). Catálogo histórico del claustro de la Universidad de San Marcos 1576-1800. Lima: Imprenta El Progreso Editorial.

Eguiguren, L. A. (1940). Diccionario Histórico Cronológico de la Real y Pontificia Universidad San Marcos. Lima: Imprenta Torres Aguirre, tomo I.

Eguiguren, L. A. (1951). La universidad Nacional Mayor de San Marcos. IV Centenario de la Fundación de la Universidad Real y Pontificia y de su vigorosa continuidad fistórica. Lima: Imprenta Santa María.

Eguiguren, L. A. (1951). La universidad en el siglo XVI, volumen I, narración. Lima: Imprenta Santa María.

Hilde de, R-S. (ed). (1999). Historia de la Universidad en Europa. 3 vol. Bilbao: Universidad del País Vasco.

Mendiburu, M. (1931-1935). Diccionario histórico biográfico del Perú. Segunda edición con adiciones y notas bibliográficas publicada por Evaristo San Cristoval. Lima: Imprenta Gil.

Mejía Valera, M. (1963). Fuentes para la historia de la filosofía en el Perú. Lima: Universidad Nacional Mayor de San Marcos.

Pretell García, M. (2015). "La Filosofía en los albores del Virreinato". Metanoia, no 1 abriloctubre, Revista Académica de la Escuela de Filosofía de la Universidad Antonio Ruiz de Montoya, pp. 75-98.

Salazar Bondy, A. (1967). La Filosofía en el Perú. Panorama histórico. Lima: Universo.

Saldaña y Flores, F. (1735). Constituciones, y Ordenanzas antiguas, añadidas, y modernas de la Real Universidad, y Estudio General de San Marcos de la Ciudad de los Reyes del Perú. Reimpressas, y recogidas de 
mandato del excelentissimo S. Marquez de Castelfuerte, Virrey. Ciudad de los Reyes, Imprenta Real.

Torchia Estrada, J. C. (1999). "Los estudios de Filosofía (Artes) en el siglo XVI: México y Perú". Revista Interamericana de Bibliografía, vol. XLIX, n $1-2$ de la Organización de Estados Americanos, pp. 69-99.
Valcárcel, D. (1953). Libro de Oposiciones del a Universidad de San Antonio del Cusco (siglo XVIII). Lima: Universidad Nacional Mayor de San Marcos.

Valcárcel, D. (1968). Historia de la Educación Colonial. Lima: Universo. 